ISSN 1980-5098 @) (1) \& DOI: http://dx.doi.org/10.5902/1980509831585

\title{
EFEITO DA TERMORRETIFICAÇÃO NA QUALIDADE DE COLAGEM DE LÂMINAS DE MADEIRA PARA A PRODUÇÃO DE COMPENSADO
}

\author{
EFFECT OF HEAT TREATMENT ON THE BONDING QUALITY OF WOOD VENEERS TO PLY- \\ WOOD PRODUCTION
}

\author{
Bruno Santos Ferreira ${ }^{1}$ Cristiane Inácio de Campos $^{2}$ Elidiane Cipriano Rangel ${ }^{3}$
}

\section{RESUMO}

O compensado é um painel de madeira que pode ser utilizado tanto na indústria moveleira quanto na construção civil, devido ao seu uso estrutural. Entretanto, para ser utilizado na construção civil, o compensado necessita ser resistente aos agentes xilófagos e à umidade, sem comprometer suas propriedades mecânicas. Nesse aspecto, a termorretificação surge como uma alternativa de tratamento, que aumenta a resistência da madeira ao ataque de fungos e a sua estabilidade dimensional. Desse modo, o objetivo deste trabalho foi avaliar o efeito da termorretificação na qualidade de colagem de lâminas de madeira para a produção de compensado. Para isso, foram realizados os testes de molhabilidade utilizando o goniômetro digital, para lâminas tratadas nas três temperaturas e na amostra de controle. Além disso, foi realizado, o ensaio de cisalhamento na linha de cola, para testar a qualidade da colagem segundo a ABNT NBR ISO 12466-1 (2006). Com os resultados obtidos pode-se perceber que a superfície da madeira tratada se tornou mais hidrofóbica com o aumento da temperatura, e isso resultou também na diminuição da resistência ao cisalhamento na linha de cola, pois o adesivo utilizado (fenol-formaldeído) é à base de água, contudo, os requisitos normativos foram atendidos.

Palavras-chave: temperatura; molhabilidade; Pinus taeda; cisalhamento na linha de cola.

\begin{abstract}
Plywood is a wood panel that can be used both in the furniture industry and in construction due to its structural use. However, to be used in construction, it needs to be resistant against wood decay agents and water, without compromising its mechanical properties. The heat treatment is an alternative treatment which improves the wood resistance to fungi and improves its dimensional stability. Thus, the objective of this study is to evaluate the influence of the heat treatment with three different temperatures, 160,180 and $200^{\circ} \mathrm{C}$ in the wettability of wood veneers and the shear strength in the bond line of the plywood produced with them. To do so, it was carried out the wettability testing using digital goniometer in the treated veneers at three temperatures and for the control specimen. Besides, it was carried out the shear strength in the bond line test, for testing the quality of bonding according to ABNT NBR ISO 12466-1 (2006). Because of the results obtained, it could be seen that the surface of the treated wood became more hydrophobic with increasing temperature, and this also resulted in a decrease in shear strength in the bond line, once the adhesive used (phenol-formaldehyde) is water based, however, the regulatory requirements have been met. Keywords: temperature; wettability; Pinus taeda; shear strenght in the bondline.
\end{abstract}

1 Engenheiro Industrial Madeireiro, MSc., Doutorando do Programa de Pós-graduação em Engenharia Mecânica, Universidade Estadual Paulista Júlio de Mesquita Filho, Av. Ariberto Pereira da Cunha, 333, Bairro Portal das Colinas, CEP 12516-410, Guaratinguetá (SP), Brasil. brunosferreira88@gmail.com

2 Engenheira Civil, Dr ${ }^{\mathrm{a}}$, Professora assistente, Universidade Estadual Paulista Júlio de Mesquita Filho, Rua Geraldo Alckmin, 519, Vila Nossa Senhora de Fátima, CEP 18409-010, Itapeva (SP), Brasil. cristiane@itapeva.unesp.br

3 Física, $\mathrm{Dr}^{\mathrm{a}}$, Professora Adjunta do Instituto de Ciência e Tecnologia de Sorocaba, Universidade Estadual PaulistaJúlio de Mesquita Filho, Av. Três de Março, 511, Bairro Alto da Boa Vista, CEP 18087-180, Sorocaba (SP), Brasil. elidiane@sorocaba.unesp.br

Recebido para publicação em 23/06/2016 e aceito em 16/11/2016

Ci. Fl., v. 28, n. 1, jan.- mar., 2018 


\section{INTRODUÇÃO}

O compensado é um exemplo de painel de madeira que pode ser utilizado tanto na produção de móveis quanto na construção civil, pois possui uso estrutural. Iwakiri (2005) define o compensado como sendo um painel fabricado através da colagem de lâminas em número ímpar de camadas, com a direção da grã perpendicular entre as camadas adjacentes.

Por possuir características estruturais é um painel utilizado em sistemas construtivos do tipo wood frame, principalmente como fechamento de teto, pisos e paredes. Entretanto, para sua utilização na construção civil no Brasil, estes painéis necessitam passar por tratamentos preservativos e de estabilização de suas propriedades, devido ao clima tropical do país, que está mais sujeito ao ataque de organismos xilófagos e ação da umidade e temperatura.

A termorretificação vem como uma alternativa de tratamento, sem utilização de compostos químicos, sendo, portanto, mais ecologicamente correta. Segundo Navi e Sandberg (2012), a termorretificação consiste em um tratamento, com a utilização apenas da temperatura, o qual promove uma degradação controlada da madeira, principalmente, das hemiceluloses, o que pode melhorar algumas de suas propriedades.

Um problema acarretado pela termorretificação, porém, é o aumento do consumo energético, já que utiliza temperaturas elevadas para o tratamento além da energia consumida no processo de secagem das lâminas e prensagem do painel. Silva (2001) em seu estudo de caso concluiu que a energia térmica consumida corresponde a $27 \%$ na etapa de laminação e $73 \%$ na fabricação do painel compensado, sendo a secagem correspondendo a $79 \%$ do consumo energético nesta etapa e a prensagem correspondendo a $2 \%$.

$\mathrm{Na}$ termorretificação, as hemiceluloses são degradadas mais rapidamente que a celulose, ou seja, possuem menor estabilidade térmica devido a sua natureza amorfa (falta de cristalinidade) e menor massa molecular quando comparadas à celulose (YILDIZ; GEZER; YILDIZ, 2006; GONZÁLEZ-PEÑA; CURLING; HALE, 2009). A degradação das hemiceluloses é mais acentuada na temperatura de $190^{\circ} \mathrm{C}$, enquanto que da celulose começa a ser mais acentuada acima de $220^{\circ} \mathrm{C}$, quando a porção cristalina da celulose passa a ser mais degradada (KAČÍKOVÁ et al., 2013).

Windeisen, Strobel e Wegener (2007) mostraram em seu estudo a diminuição dos grupos hidroxílicos alifáticos, durante a termorretificação, ocorrendo principalmente, na temperatura mais elevada de $200^{\circ} \mathrm{C}$. Nesta temperatura, a quantidade de grupos hidroxílicos alifáticos foi reduzida para $60 \%$ se comparada à madeira sem termorretificação. Esta diminuição é devido às reações de oxidação e desidratação que ocorrem nestas temperaturas mais elevadas.

Teoricamente, a disponibilidade dos grupos $\mathrm{OH}$ das hemiceluloses tem o efeito mais significativo nas propriedades físicas da madeira. $\mathrm{O}$ tratamento térmico diminui a captação de água e a parede celular absorve menos água pela diminuição da quantidade de grupos hidroxila. Como consequência da redução dos grupos hidroxila, o inchamento e a retração são menores (GÜNDÜZ; KORKUT; KORKUT, 2008).

A principal característica da termorretificação na madeira é a melhora na sua estabilidade dimensional. Isto pode ser explicado devido à degradação das hemiceluloses, que ocorre através de reações de desidratação com a destruição dos grupos hidroxila. Portanto, a degradação das hemiceluloses, que são os polímeros mais hidrofílicos da madeira, devido a sua estrutura amorfa e grande quantidade de grupos hidroxila, diminui a afinidade da madeira com a água e aumenta a estabilidade dimensional (WEILAND; GUYONNET, 2003).

Tjeerdsma et al. (1998) também explicam que a redução da higroscopicidade e, consequente, melhoria da estabilidade dimensional também se devem a uma macromolécula mais firme e inelástica de lignina, que passou por reações de reticulação e condensamento após a termorretificação. As moléculas de lignina rodeiam as microfibrilas de celulose, e como sua estrutura está mais rígida, faz com que a capacidade de expansão das microfibrilas de celulose diminua e, consequentemente, sua capacidade de absorver água.

Outra propriedade da madeira que apresenta mudança com a termorretificação é a rugosidade superficial. Em um estudo realizado por Kamdem, Pizzi e Jermannaud (2002), a redução da rugosidade superficial da madeira tratada termicamente foi significativa. Unsal e Ayrilmis (2005) mostraram que a rugosidade superficial da madeira de Eucalyptus camaldulensis diminuiu com o aumento da temperatura e do tempo de termorretificação, chegando a uma diminuição de $27,9 \%$ da rugosidade para a temperatura de 
$180^{\circ} \mathrm{C}$ com um tratamento de 10 horas. Outros autores como Salca e Hiziroglu (2014) e Günduz, Korkut e Korkut (2008) também chegaram à mesma conclusão para diferentes espécies de madeira, ou seja, o aumento da temperatura e do tempo do tratamento térmico diminui a rugosidade superficial para diferentes espécies de madeira.

Apesar da melhoria das propriedades físicas, vários autores relatam a diminuição das propriedades mecânicas da madeira com o aumento do tempo e da temperatura de termorretificação (YILDIZ; GEZER; YILDIZ, 2006; KORKUT; AKGÜL; DÜNDAR, 2008; KORKUT et al., 2008; GUNDUZ; AYDEMIR; KARAKAS, 2009; PONCSAK; KOCAEFE; YOUNSI, 2011; KAČÍKOVÁ et al., 2013; SALCA; HIZIROGLU, 2014).

A molhabilidade, que é uma propriedade que avalia a interação entre um líquido e a uma superfície através da medição do ângulo de gota, também sofre influência da termorretificação. Gérardin et al. (2007) afirmam que a molhabilidade da madeira, após tratamento térmico, é menor para solventes polares e maior para solventes apolares. Soares et al. (2011) mostraram que a molhabilidade diminuiu com o aumento da temperatura até $200^{\circ} \mathrm{C}$ e foi influenciada pelo tempo de tratamento, tipo de amostra e região de corte da madeira. Hakkou et al. (2005) concluíram que a maior influência da diminuição da molhabilidade é a plastificação da lignina ocorrida com o aumento da temperatura, afetando particularmente as propriedades hidrofílicas da madeira. Com a diminuição da molhabilidade ocorre, consequentemente, a diminuição na qualidade de colagem.

No entanto, a termorretificação aumenta a resistência da madeira ao ataque de fungos, pois há formação de compostos que agem como fungicidas. Estes compostos são derivados da decomposição térmica da celulose, mas, principalmente, das hemiceluloses, que são decompostas em temperaturas mais baixas (WEILAND; GUYONNET, 2003). Outros autores confirmam esta tese e afirmam também que o simples fato de decompor as hemiceluloses já contribui muito para a redução do ataque de fungos, pois são as moléculas da madeira de mais fácil digestão para os fungos (TJEERDSMA et al., 1998; HAKKOU et al., 2006; BOONSTRA et al., 2007; WINDEISEN; STROBEL; WEGENER, 2007; BROSSE et al., 2010).

Devido a esta capacidade de melhoria na estabilidade dimensional e na resistência ao ataque de fungos, é que surge este estudo, com o intuito de realizar a termorretificação em painéis de compensado a serem utilizados em sistemas construtivos do tipo wood frame como fechamento de paredes, tetos e pisos. Portanto, este estudo tem como objetivo avaliar o efeito da termorretificação na qualidade de colagem de lâminas de madeira para a produção de compensado, utilizando o teste de cisalhamento na linha de cola e de molhabilidade das lâminas.

\section{MATERIAIS E MÉTODOS}

Neste estudo foi realizada a termorretificação das lâminas de madeira, antes da produção do painel compensado. Para a análise da termorretificação foram realizados os tratamentos nas temperaturas de $160^{\circ} \mathrm{C}(\mathrm{T} 160), 180^{\circ} \mathrm{C}(\mathrm{T} 180)$ e $200^{\circ} \mathrm{C}(\mathrm{T} 200)$, além disso, foram produzidos compensados sem tratamento para serem utilizados como controle (T0). Para cada tratamento foram produzidos quatro painéis. Foram escolhidas estas temperaturas, pois acima de $200^{\circ} \mathrm{C}$, como exposto anteriormente, há uma degradação elevada da celulose, o que acarretaria em grande diminuição das propriedades mecânicas (MENDES, 2010; SILVA, 2012). Para cada tratamento foram produzidos no total quatro painéis.

\section{Metodologia da produção dos painéis}

Os painéis compensados foram produzidos com lâminas de Pinus taeda, provenientes da região de Imbituva - PR com idade de 20 anos e DAP em torno de 28 e $32 \mathrm{~cm}$, conforme informação fornecida pela empresa doadora do material, seguindo os passos descritos abaixo:

- Secagem das lâminas com espessura de 2,3 mm em uma estufa laboratorial até estabilização do teor de umidade a $3 \%$.

- Preparação e aplicação do adesivo à base de fenol-formaldeído, na gramatura de $395 \mathrm{~g} / \mathrm{m}^{2}$ linha dupla. Este adesivo foi preparado com uma mistura de resina fenol-formaldeído, farinha de trigo e água nas 
proporções de 100:10:10, respectivamente. A resina utilizada do tipo Cascophen HL 7090 com 62\% de teor de sólidos, $\mathrm{pH}$ igual a 11 e viscosidade de $415 \mathrm{cP}$.

- Sobreposição de 7 lâminas, dispostas de forma perpendicular à lâmina adjacente, resultando em uma espessura nominal do compensado de $16 \mathrm{~mm}$.

- Pré-prensagem a frio, em uma prensa manual com pressão de $1 \mathrm{kgf} / \mathrm{cm}^{2}$, para retirada de excesso de ar entre as lâminas, com a intenção de eliminar a formação de bolhas durante o processo de prensagem a quente.

- Prensagem a quente com pressão de $10 \mathrm{kgf} / \mathrm{cm}^{2}$, temperatura de $180^{\circ} \mathrm{C}$ por 10 minutos, dividida em três ciclos de prensagem com 3 minutos cada e 30 segundos de alívio da pressão entre os ciclos.

\section{Termorretificação}

A termorretificação das lâminas foi realizada em estufa laboratorial sem substituição de ar. Para isso, primeiramente, as lâminas foram empilhadas dentro da estufa com a utilização de tabiques entre elas, com o intuito de que todas as superfícies ficassem em contato direto com a temperatura. Após isso, iniciouse o processo de aquecimento a uma taxa de $2,6^{\circ} \mathrm{C} / \mathrm{min}$, até atingir a temperatura especificada para cada tratamento $\left(160^{\circ} \mathrm{C}, 180^{\circ} \mathrm{C}\right.$ e $\left.200^{\circ} \mathrm{C}\right)$. Sendo, o tempo de aquecimento aproximadamente de 52 minutos para T160, 60 minutos para T180 e 68 minutos para T200. Quando se atingiu a temperatura necessária, iniciou-se o processo de termorretificação durante 60 minutos para todos os tratamentos. A estufa então foi desligada, e as lâminas permaneceram em seu interior por mais 30 minutos até o resfriamento e foram levadas imediatamente para a produção dos painéis.

\section{Teste de Molhabilidade das lâminas}

Para o teste de molhabilidade da lâmina foi realizada a medição do ângulo de gota sobre a superfície das lâminas. Para a realização deste teste foi utilizado um goniômetro digital (Figura 1), e como líquido para a formação da bolha utilizou-se a água deionizada. As amostras selecionadas possuíam dimensões de 10 × $35 \mathrm{~mm}$ com espessura de $2,3 \mathrm{~mm}$, a própria espessura da lâmina. Para cada tratamento foram depositadas três gotas na superfície e após 10 segundos de acomodação, foi realizada a medição do ângulo, cada medição resultou em 10 valores.

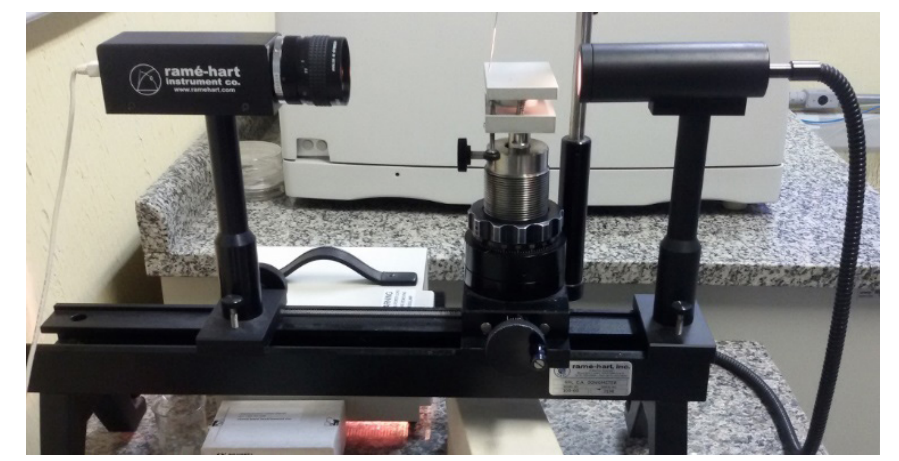

FIGURA 1: Goniômetro digital utilizado no teste de molhabilidade.

FIGURE 1: Digital goniometer used in the wettability test.

\section{Ensaio de cisalhamento na linha de cola}

Os ensaios de cisalhamento na linha de cola foram realizados de acordo com a norma da ABNT NBR ISO 12466-1 (2006). Primeiramente, foram confeccionados os corpos de prova com dimensões de $150 \times 25 \times 16 \mathrm{~mm}$, nos quais foram realizados dois sulcos distantes um do outro por $25 \mathrm{~mm}$ (Figura 2). 


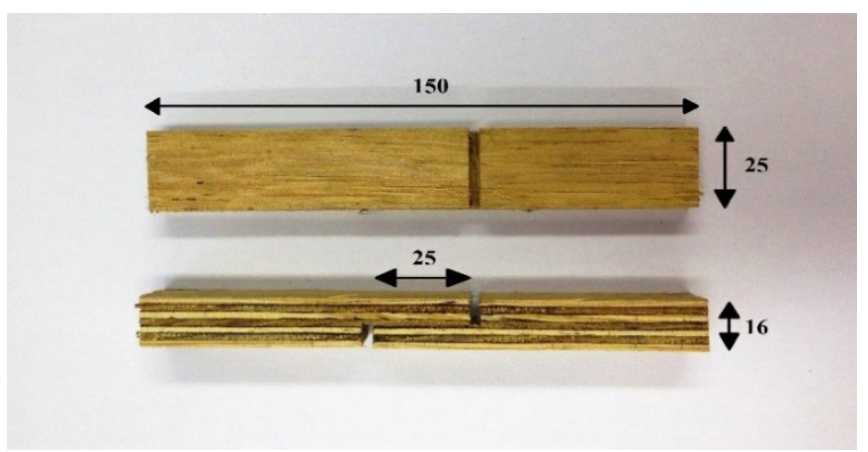

FIGURA 2: Corpo de prova de cisalhamento da linha de cola, valores em milímetros. FIGURE 2: Shear strength in the bondline specimen, measured in millimetres.

Os ensaios foram realizados na máquina universal de ensaios da marca EMIC - capacidade de carga de 30 toneladas com velocidade de carregamento de 120 N/s. Foram ensaiadas 6 amostras por tratamento.

\section{ANÁLISE ESTATÍSTICA}

Os resultados obtidos foram avaliados pela análise de regressão linear simples, em um nível de significância de 5\% para a análise de variância da regressão. Para tal, foram verificadas inicialmente as condicionantes de regressão que são a normalidade dos dados e a homogeneidade das variâncias, através dos testes de Shapiro Wilk e Bartlett, respectivamente. Todas as análises foram realizadas no software R versão 3.2.3 de 2015 .

\section{RESULTADOS E DISCUSSÃO}

Para o teste de molhabilidade, as médias dos resultados encontrados, juntamente com o desvio padrão, estão apresentadas na Tabela 1 .

TABELA 1: Valores médios encontrados para o ângulo de gota em cada tratamento.

TABLE 1: Average values found for the drop angle of each treatment.

\begin{tabular}{ccccc}
\hline & \multicolumn{2}{c}{ Ângulo da gota } \\
T0 & T160 & T180 & T200 \\
\hline Média & 39,88 & 73,48 & 93,83 & 96,08 \\
DP & 2,18 & 3,10 & 3,12 & 0,83 \\
CV & $5,46 \%$ & $4,21 \%$ & $3,33 \%$ & $0,86 \%$ \\
\hline
\end{tabular}

Em que: DP = Desvio Padrão; CV = Coeficiente de Variação.

Através da análise de variância da regressão $\left(\mathrm{F}_{\text {value }}=530.5\right.$; p-value $\left.=2,2 \times 10^{-16}<0,05\right)$ pôde-se notar que houve diferença significativa entre os tratamentos, evidenciando que o aumento da temperatura aumenta o ângulo da gota, diminuindo, portanto, a molhabilidade da superfície, ou seja, a melhor molhabilidade ocorreu para o tratamento T0, no qual se obteve o menor ângulo de contato. Isso mostra que a termorretificação e o aumento da temperatura tornam a superfície mais hidrofóbica, isso pode influenciar na colagem do painel compensado, pois o adesivo utilizado fenol-formaldeído é um adesivo à base de água. A Figura 3 apresenta o gráfico da regressão linear, com coeficiente de determinação $\mathrm{R}^{2}=0,87$. 


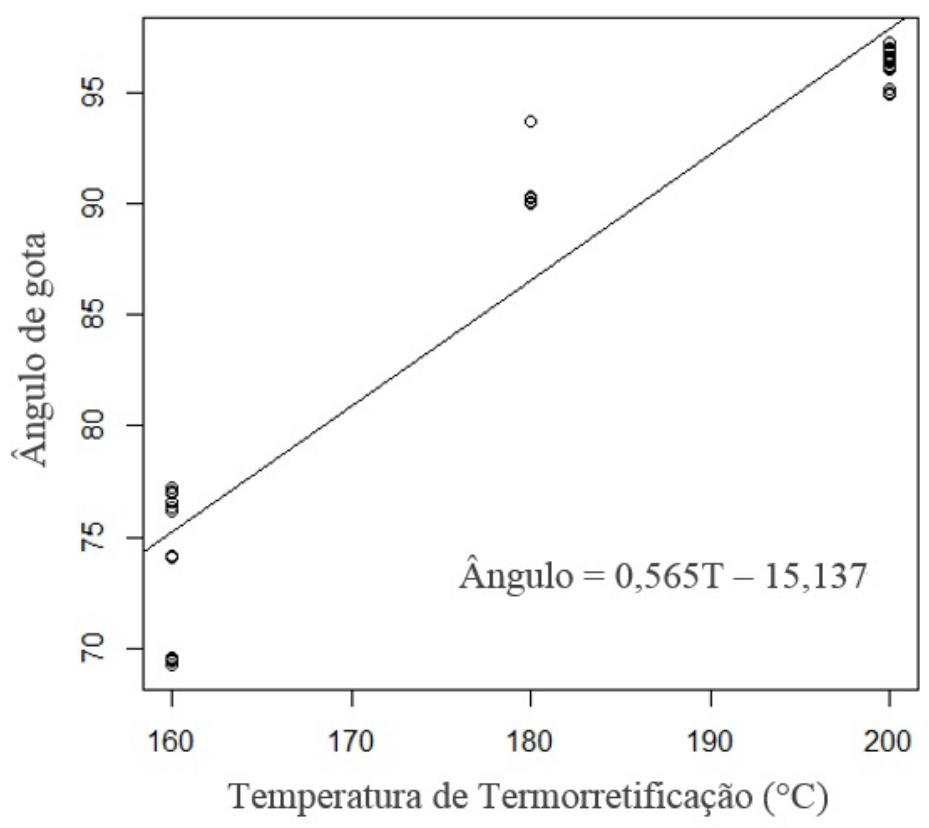

FIGURA 3: Gráfico da regressão linear do ângulo de gota em função da temperatura de termorretificação. FIGURE 3: Graph of linear regression of the drop angle as a function of heat treatment temperature.

$\mathrm{O}$ valor do ângulo de gota para o tratamento T0 foi cerca de $45,72 \%$ menor se comparado ao tratamento T160 e 58,49\% menor se comparado ao tratamento T200, mostrando que a penetração e a fluidez da gota sobre a superfície sem tratamento foram maiores. Isso evidencia que a termorretificação diminui a penetração e a fluidez da água e de qualquer outra substância hidrofílica. A Figura 4 apresenta as gotas obtidas no goniômetro digital para cada tratamento.

(a)

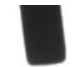

(b)

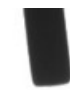

(c)

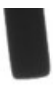

(d)
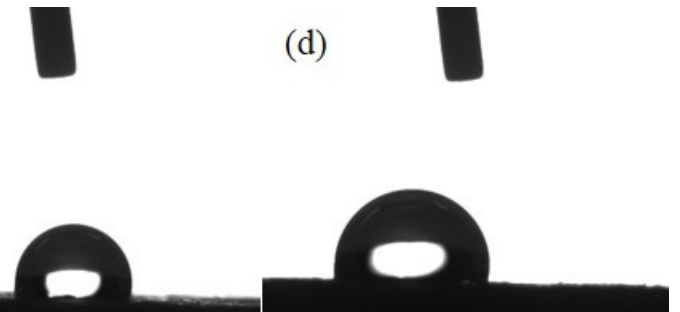

FIGURA 4: Gotas obtidas para cada tratamento; (a) sem temperatura; (b) $160^{\circ} \mathrm{C}$; (c) $180^{\circ} \mathrm{C}$; (d) $200^{\circ} \mathrm{C}$.

FIGURE 4: Drops obtained for each treatment; (a) without heat treatment; (b) $160^{\circ} \mathrm{C}$; (c) $180^{\circ} \mathrm{C}$; (d) $200^{\circ} \mathrm{C}$.

Gérardin et al. (2007) concluíram em seu estudo que a molhabilidade após o tratamento térmico se torna menor para solventes polares e maior para solventes apolares. No presente estudo, isso foi comprovado, com os resultados apresentados na Tabela 1, pois o liquido utilizado para o teste de molhabilidade foi a água deionizada, que é um solvente polar. Ainda quanto ao efeito da termorretificação na molhabilidade, Hakkou et al. (2005) concluíram que a plastificação da lignina provocada pela elevação da temperatura torna-se a maior interferência na redução da molhabilidade da madeira com líquidos hidrofílicos, evidenciado também no presente trabalho.

A Tabela 2 apresenta os valores médios e os desvios padrões obtidos no teste de cisalhamento na linha de cola para todos os tratamentos. 
TABELA 2: Resultados médios obtidos no teste de cisalhamento na linha de cola.

TABLE 2: Average results obtained in the shear stress in the bondline.

\begin{tabular}{lcccc}
\hline & \multicolumn{4}{c}{ Cisalhamento na linha de cola (MPa) } \\
& T0 & T160 & T180 & T200 \\
\hline Média & 3,75 & 3,27 & 2,77 & 2,31 \\
DP & 0,47 & 0,16 & 0,19 & 0,20 \\
CV & $12,56 \%$ & $4,86 \%$ & $7,04 \%$ & $8,47 \%$ \\
\hline
\end{tabular}

Em que: DP = Desvio Padrão; CV = Coeficiente de Variação.

Através da análise de variância da regressão $\left(F_{\text {value }}=88,49\right.$; p-value $\left.=6,39 \times 10^{-8}<0,05\right)$ pôdese notar que houve diferença significativa entre os tratamentos. Portanto, o aumento da temperatura de termorretificação causou a diminuição da resistência ao cisalhamento na linha de cola. Isso pode ser justificado pelo fato de também ter ocorrido a diminuição da molhabilidade da superfície, pois ela se tornou mais hidrofóbica, diminuindo a interação entre a superfície da lâmina e o adesivo fenol-formaldeído, que é hidrossolúvel. A Figura 5 apresenta o gráfico da regressão linear, com coeficiente de determinação $\mathrm{R}^{2}=$ 0,85 .

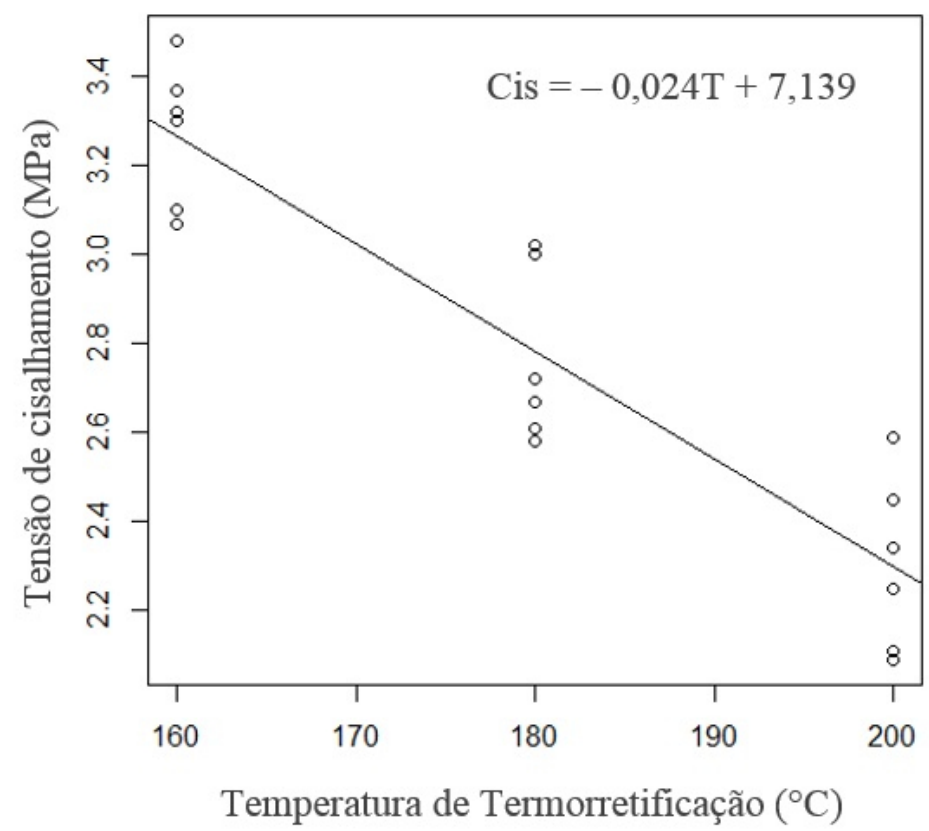

FIGURA 5: Gráfico da regressão linear da tensão de cisalhamento na linha de cola em função da temperatura de termorretificação.

FIGURE 5: Graph of the linear regression of the shear strenght in the bond line as a function of heat treatment temperature.

Pelos resultados obtidos pode-se notar que a termorretificação diminuiu a molhabilidade da superfície e, consequentemente, diminuiu a resistência ao cisalhamento na linha de cola do painel de compensado. Contudo, ainda o valor de cisalhamento no tratamento mais intenso T200 é superior ao exigido pela norma ABNT NBR ISSO 12466-2 (2006) que é de $1 \mathrm{MPa}$. O gráfico da Figura 6 apresenta os valores obtidos de tensão de cisalhamento comparados ao valor de referência.

Pelo gráfico da Figura 6 pode-se notar a queda de resistência das três temperaturas quando comparada ao tratamento T0. A maior diferença ocorreu na temperatura de $200^{\circ} \mathrm{C}$, na qual houve a menor qualidade de colagem e menor molhabilidade, ou seja, maior ângulo da gota. Windeisen, Strobel e Wegener (2007) apresentaram em seu estudo, que na termorretificação a $200^{\circ} \mathrm{C}$ ocorreu uma diminuição de $60 \%$ na quantidade de grupos hidroxílicos alifáticos, isso justifica a diminuição de molhabilidade e, consequentemente, a diminuição na resistência ao cisalhamento, pois torna a superfície mais hidrofóbica. 
A termorretificação ocasiona a diminuição na resistência ao cisalhamento, entretanto, esta diminuição até à temperatura de $200^{\circ} \mathrm{C}$ se mostrou muito acima do exigido pela especificação normativa. Também pelos resultados obtidos pode-se perceber que o aumento da temperatura de termorretificação torna a superfície menos hidrofílica, o que proporcionará melhor estabilidade dimensional para o painel produzido.

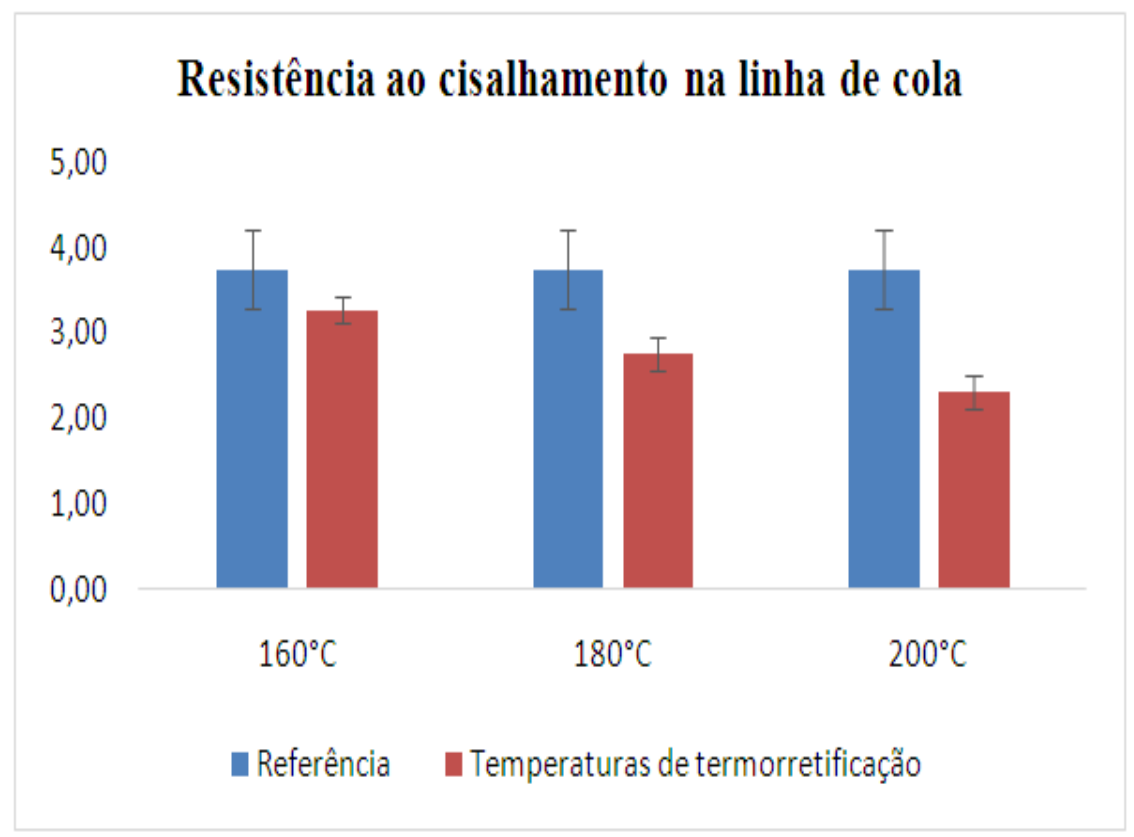

FIGURA 6: Gráfico de comparação entre os valores de resistência ao cisalhamneto da linha de cola da referência com as três temperaturas de termorretificação.

FIGURE 6: Comparison graph between the shear strenght in the bondline values of the reference and the three heat treatment temperatures.

\section{CONCLUSÕES}

Pode-se concluir, com este trabalho, que a termorretificação diminuiu a molhabilidade da superfície da lâmina e, consequentemente, prejudicou a qualidade de colagem reduzindo a resistência ao cisalhamento na linha de cola, devido à menor interação entre o adesivo, que é hidrofílico, e a lâmina de madeira termorretificada. Destaca-se que, embora tenha ocorrido esta redução, especialmente para o tratamento T200, os limites normativos de no mínimo $1 \mathrm{MPa}$ continuaram sendo atendidos, o que indica a viabilidade dos três tratamentos estudados. Ressalta-se ainda, que o principal aspecto negativo da termorretificação é o aumento no consumo energético, fator que deve ser estudado em trabalhos futuros, no entanto, existem sistemas computacionais que otimizam o consumo energético no processo de secagem de lâminas e termorretificação e que podem viabilizar tal tratamento.

\section{AGRADECIMENTOS}

Agradecemos à CAPES pelo apoio financeiro para a realização desta pesquisa.

\section{REFERÊNCIAS}

ASSOCIAÇÃO BRASILEIRA DE NORMAS TÉCNICAS. NBR ISO 12466-1: Compensado: determinação da massa específica aparente. Rio de Janeiro, 2006.

ASSOCIAÇÃO BRASILEIRA DE NORMAS TÉCNICAS. NBR ISO 12466-2: Compensado: determinação da massa específica aparente. Rio de Janeiro, 2006.

BOONSTRA, M. J. et al. Optimisation of a two-stage heat treatment process: durability aspects. Wood 
Science and Technology, München, v. 41, n. 1, p. 31-57, 2007.

BROSSE, N. et al. Investigation of the chemical modifications of beech wood lignin during heat treatment. Polymer Degradation and Stability, Aubière, v. 95, n. 9, p. 1721-1726, 2010.

GÉRARDIN, P. et al. Evolution of wood surface free energy after heat treatment. Polymer Degradation and Stability, Aubière, v. 92, n. 4, p. 653-657, 2007.

GONZÁLEZ-PEÑA, M. M.; CURLING, S. F.; HALE, M. D. C. On the effect of heat on the chemical composition and dimensions of thermally-modified wood. Polymer Degradation and Stability, Aubière, v. 94, n. 12, p. 2184-2193, 2009.

GUNDUZ, G.; AYDEMIR, D.; KARAKAS, G. The effects of thermal treatment on the mechanical properties of wild Pear (Pyrus elaeagnifolia Pall.) wood and changes in physical properties. Materials and Design, Oxford, v. 30, n. 10, p. 4391-4395, 2009.

GÜNDÜZ, G.; KORKUT, S.; KORKUT, D. S. The effects of heat treatment on physical and technological properties and surface roughness of Camiyani Black Pine (Pinus nigra Arn. subsp. pallasiana var. pallasiana) wood. Bioresource technology, Mohali, v. 99, n. 7, p. 2275-2280, 2008.

HAKKOU, M. et al. Investigation of wood wettability changes during heat treatment on the basis of chemical analysis. Polymer Degradation and Stability, Aubière, v. 89, n. 1, p. 1-5, 2005.

HAKKOU, M. et al. Investigations of the reasons for fungal durability of heat-treated beech wood. Polymer Degradation and Stability, Aubière, v. 91, n. 2, p. 393-397, 2006.

IWAKIRI, S. Painéis de madeira reconstituída. Curtiba: FUPEF, 2005. 247 p.

KAČÍKOVÁ, D. et al. Effects of thermal treatment on chemical, mechanical and colour traits in Norway spruce wood. Bioresource Technology, Mohali, v. 144, p. 669-674, 2013.

KAMDEM, D. P.; PIZZI, A.; JERMANNAUD, A. Durability of heat-treated wood. Holz als Roh - und Werkstoff, München, v. 60, n. 1, p. 1-6, 2002.

KORKUT, S.; AKGÜL, M.; DÜNDAR, T. The effects of heat treatment on some technological properties of Scots pine (Pinus sylvestris L.) wood. Bioresource Technology, Mohali, v. 99, n. 6, p. 1861-1868, 2008. KORKUT, S. et al. The effects of heat treatment on technological properties in Red-bud maple (Acer trautvetteri Medw.) wood. Bioresource Technology, Mohali, v. 99, n. 6, p. 1538-1543, 2008.

NAVI, P.; SANDBERG, D. Heat Treatment. In: NAVI, P.; SANDBERG, D. Thermo-Hydro-Mechanical processing of wood. Boca Raton: CRC Press, 2012. cap. 9.

MENDES R. F. Efeito do tratamento térmico sobre painéis OSB. 2010. 116 f. Dissertação (Mestrado em Recursos Florestais) - Universidade de São Paulo, Piracicaba, 2010.

PONCSAK, S.; KOCAEFE, D.; YOUNSI, R. Improvement of the heat treatment of Jack pine (Pinus banksiana) using ThermoWood technology. European Journal of Wood and Wood Products, München, v. 69, n. 2, p. 281-286, 2011.

SALCA, E. A.; HIZIROGLU, S. Evaluation of hardness and surface quality of different wood species as function of heat treatment. Materials and Design, Oxford, v. 62, p. 416-423, 2014.

SILVA, D. A. Avaliação da eficiência energética em uma indústria de painéis compensado. 2001. 205 f. Tese (Doutorado em Ciências Florestais) - Universidade Federal do Paraná, Curitiba, 2001.

SILVA, M. R. Efeito do tratamento térmico nas propriedades químicas, físicas e mecânicas em elementos estruturais de Eucalipto citriodora e Pinus taeda. 2012. $223 \mathrm{f}$. Tese (Doutorado em Ciência e Engenharia dos Materiais) - Universidade de São Paulo, São Carlos, 2012.

SOARES, A. C. et al. Molhabilidade em amostras de Araucaria angustifolia e Pinus elliottii após tratamento térmico e envelhecimento. Scientia Forestalis, Piracicaba, v. 39, n. 92, p. 447-456, 2011.

TJEERDSMA, B. F. et al. Characterisation of thermally modified wood: molecular reasons for wood performance improvement. Holz als Roh- und Werkstoff, München, v. 56, p. 149-153, 1998.

UNSAL, O.; AYRILMIS, N. Variations in compression strength and surface roughness of heat-treated Turkish river red gum (Eucalyptus camaldulensis) wood. Journal of Wood Science, Uji, v. 51, n. 4, p. 405-409, 2005.

WEILAND, J. J.; GUYONNET, R. Study of chemical modifications and fungi degradation of thermally modified wood using DRIFT spectroscopy. Holz als Roh- und Werkstoff, München, v. 61, p. 216-220, 2003.

WINDEISEN, E.; STROBEL, C.; WEGENER, G. Chemical changes during the production of thermotreated beech wood. Wood Science and Technology, München, v. 41, n. 6, p. 523-536, 2007.

YILDIZ, S.; GEZER, E. D.; YILDIZ, U. C. Mechanical and chemical behavior of spruce wood modified by heat. Building and Environment, West Lafayette, v. 41, n. 12, p. 1762-1766, 2006. 